\title{
La relación universidad - empresa a la luz del desarrollo de las prácticas Pre - Profesionales
}

\author{
PhD. Maribel Asín Cala \\ Universidad de Oriente \\ maribela@uo.edu.cu
}

\author{
PhD. Daniel Fuentes Almaguer \\ Universidad de Oriente \\ daniel.fuentes@uo.edu.ec
}

Palabras claves: formación del profesional, práctica laboral investigativa,
tutoría

Keywords: professional training, investigative work practice, mentoring.
Recibido: 13 de abril de 2017

Aceptado: 11 de julio de 2017

\section{RESUMEN}

La formación de profesionales, como cualquier otra función de la educa- ción superior, está relacionada con procesos sociales más generales y cons- tituye una función que está determinada por los propios objetivos del sistema socioeconómico y político en que se encuentra insertada, de la cual es pro- ducto y agente de su desarrollo; por lo que está estructurada y obedece al di- seño de un conjunto de relaciones sociales que entrelazan diversos com- ponentes e instituciones de una situación social dada. Es propósito de este artículo reflexionar acerca de las potencialidades y oportunidades del víncu- lo universidad- empresa en la formación de los profesionales competentes.

\begin{abstract}
The formation of professionals, like any other show of higher education, she is related with social processes more general and constitute a show that she is determined for the very objectives of the socioeconomic system and politician in which one finds itself inserted, that of whom is product and factor of his development in; What you are in favor of structured and obey the designing of a set of social relations that various components and institutions of a social given situation intertwine. Purpose comes from this article to reflect about potentialities and opportunities of the link university company in the competent professionals' formation.
\end{abstract}




\section{INTRODUCCIÓN}

La universidad en su devenir histórico, a tono con el carácter socio-histórico y clasista de la educación, se ha ido transformando y adaptando a las necesidades y exigencias sociales, observándose una relación dialéctica en este proceso transformador. Las nuevas condiciones socioeconómicas y científicas técnicas le exigen a la universidad una necesaria transformación, tanto desde el punto de vista de sus concepciones como de sus prácticas educativas. Para lograrlo la universidad necesita perfeccionar los principales procesos que en ella se desarrollan.

En la actualidad el perfeccionamiento de la planificación, organización, desarrollo, control y evaluación de la formación en los contextos laborales (prácticas laborales o pre-profesionales) constituye una necesidad y un reto para las instituciones formadoras de nivel superior.

Es importante que el centro formador, los estudiantes y las empresas e instituciones que acogen a los estudiantes durante las prácticas comprendan su papel:

- los estudiantes: que las prácticas laborales o pre -profesionales constituyen un espacio para su aprendizaje y perfeccionamiento profesional,

- las empresas e instituciones: comprendan que las prácticas laborales o preprofesionales constituyen una oportunidad para contribuir con el desarrollo de la comunidad, de la sociedad y de la propia empresa o institución,

- la institución formadora: que el desarrollo de las prácticas pre- profesionales debe favorecer el desarrollo de la estrategia de formación de los profesionales competentes, en la cual deben estar involucrados que todos sus agentes formadores y los estudiantes.

Sin embargo, todavía se aprecian insuficiencias en el vínculo universidad - empresa en función del desarrollo del profesional durante la formación inicial. Lo anterior exige fortalecer el trabajo que realizan las universidades, empresas e instituciones en la formación del profesional, lograr mayor vinculación de los profesores universitarios con los procesos que se desarrollan en los escenarios donde los estudiantes realizaran la práctica laboral y de los especialistas de la producción y los servicios en la formación de pregrado.

Es propósito de este trabajo ofrecer algunas reflexiones sobre la necesidad de fortalecer el vínculo universidad- empresa en función de perfeccionar el desarrollo de la práctica laboral investigativa y contribuir a la formación de profesionales competentes.

\section{DESARROLLO}

En el mundo actual la vinculación universidad - empresa adquiere particular importancia, aunque es válido reconocer que no es un fenómeno nuevo ni particular de América Latina ni de los países más desarrollados. En reiteradas conferencias y 
reuniones, la UNESCO ha reiterado la necesidad de que la Universidad establezca vínculos con unidades de la producción y los servicios para así contribuir al desarrollo tecnológico y a identificar las necesidades de las empresas.

Para cumplir con este propósito es necesario que la universidad se acerque más a los procesos que se desarrollan en las diferentes empresas e instituciones socioeconómicas, que identifiquen sus necesidades y adecuen los programas y cursos de la formación inicial y post graduada a sus requerimientos. Ello supone poner un mayor énfasis en la enseñanza, no solo como una misión universitaria en sí misma, sino también como base para articular las relaciones entre la universidad y su entorno socioeconómico. Una vía para lograrlo lo constituye la vinculación del estudio con el trabajo, particularmente - en este caso- a través de las prácticas laborales o pre profesionales.

La vinculación del estudio con el trabajo, de la teoría con la práctica y con la vida, constituye uno de los principios rectores, no solo de la educación cubana, sino de la educación en diferentes países del mundo. Su esencia está en las ideas educativas y pedagógicas de destacados educadores. Quizás su antecedente más conocido está en la teoría pedagógica del destacado pedagogo checo Juan Amus Comenius (15921670) cuando expresó que "lo que ha de hacerse, debe aprenderse haciéndolo".

El más universal de los cubanos José Martí (1853-1895) consideraba que la práctica laboral es sostén para situar al hombre en la realidad de su mundo, basado en el hecho de que: "Ventajas físicas, mentales y morales vienen del trabajo...El hombre crece con el trabajo que sale de sus manos". Más cercano en el tiempo Fidel Castro (1926-2016) expresó: “...no puede haber estudio sin vinculación al trabajo, no puede haber formación sin vinculación a las actividades productivas, no se puede concebir al hombre como un intelectual en abstracto, ajeno al mundo en que vive...". Tales planteamientos constituyen las bases de las prácticas laborales investigativas en la universidad.

Es por ello que en el proceso de enseñanza aprendizaje de todas las asignaturas, se aprecian dos componentes: uno teórico y otro práctico, componentes que deben ser integrados para lograr la efectividad de la enseñanza y del aprendizaje. El Dr. Carlos Álvarez, en su libro "La escuela en la vida", hace referencia a estos dos componentes, señalando que en la concepción de toda asignatura se incluye no sólo lo académico sino también lo laboral y que es tan importante lo conceptual como las habilidades que se desarrollan para la solución de problemas de la práctica social, y que ninguno de estos elementos del proceso docente educativo puede considerarse relegados a un segundo plano. Pero no sólo en las clases o a través del contenido de las asignaturas se logra el enfoque laboral, éste se pone de manifiesto en todas las actividades de la vida de las instituciones educativas.

En el caso de la educación superior la relación del estudio con el trabajo adquiere características sui generis porque debe establecer de forma prioritaria una relación obligada y con carácter de ley entre la misión de la Universidad y las demandas 
sociales que se corresponden con ésta en cada momento histórico, social y cultural concreto. Es por ello que la preocupación por la calidad de los estudios arranca de la idea de que los estudiantes de la educación superior de un país forman parte de la riqueza de ese país, incluso en el futuro, las universidades van a ser juzgadas más por la calidad de sus alumnos que por la de sus profesores.

Así, por ejemplo, en el Patrón de Evaluación y Acreditación de los Centros de Educación Superior, carreras y figuras del postgrado, un aspecto que se evalúa en diferentes variables es el vínculo con el territorio, sus empresas y unidades de servicios, así como el desarrollo de la práctica laboral. La importancia de la relación de la Universidad con las empresas aparece refrendada en los acuerdos e informes de diferentes foros de organismos como la UNESCO y la CEPAL. Se plantea por ejemplo que: "Existen diversos factores que pueden facilitar la creación y el fortalecimiento de la interrelación entre las universidades y las empresas (elementos de convergencia) y otros que, por el contrario, pueden dificultar o entorpecer dicha vinculación (elementos de divergencia)".

- $\quad$ Elementos de convergencia: las universidades y las empresas son actores primarios en los procesos de innovación y ambos poseen habilidades y capacidades científicas y tecnológicas fundamentales para la generación de conocimiento, que muchas veces resultan complementarias.

- $\quad$ Elementos de divergencia: a pesar de compartir la particularidad de desarrollar y utilizar capacidades para la generación de conocimiento científico-tecnológico, la universidad y la empresa llevan adelante esta tarea con competencias, finalidades y criterios muy distintos.

Por tanto, la creación de vínculos entre universidades y empresas debe desarrollarse según la noción de especialización complementaria: generar y fortalecer los factores de convergencia entre ellas, es decir, sus respectivas capacidades científicas y tecnológicas y, al mismo tiempo, reducir los elementos de divergencia, sin que esto implique eliminar las especificidades, objetivos y misiones de cada una.

Este vínculo se manifiesta de diferentes formas y se establece a través de diferentes canales, obteniéndose diferentes beneficios. Por ejemplo: flujos de recursos humanos (por ejemplo, las prácticas de los estudiantes en empresas o la contratación de nuevos profesionales), las redes informales entre profesionales, las actividades de difusión del conocimiento (conferencias, elaboración de publicaciones), los proyectos específicos, los servicios de asesoramiento y apoyo tecnológico (asistencia técnica, equipo técnico), el licenciamiento de tecnología (patentes, oficinas de transferencia tecnológicas de las universidades) y la creación de centros mixtos o empresas de base tecnológica.

En el caso de Cuba, lograr tal propósito, implica que la universidad establezca sólidas relaciones con los Organismos de la Administración Central del Estado (OACE) y dentro de ellos con aquellas entidades, empresas, organismos e instituciones que puedan contribuir de manera exitosa a la formación del profesional, es decir con las 
Unidades Docentes (UD) y Entidades Laborales de Base (ELB), centros donde de manera planificada se realizan las prácticas laborales.

La práctica laboral, concebida como forma específica de organización del proceso docente educativo en los centros de educación superior cubanos, constituye una vía eficaz para la activación de las diferentes esferas de la personalidad y contribuye a desarrollar cualidades morales. Por otro lado, la incorporación de los alumnos a las prácticas laborales en diferentes empresas e instituciones contribuye de forma decisiva en la formación de capacidades de actuación y de inserción social, a desarrollar una actitud positiva y de respeto al trabajo, a dominar los procedimientos en la resolución de problemas, a saber aplicar y combinar los conocimientos específicos y procedimientos generales en la solución de problemas de la vida, a realizar una mayor valoración de la actividad creativa, a comprender la incidencia de los avances científico-técnicos sobre las formas de vida y a desarrollar la capacidad de decisión.

Se comparte la idea expresada por Herrera (2006) referida a que la práctica investigativo-laboral es un proceso de profesionalización porque como proceso educativo influye en el crecimiento económico y el bienestar material, puede ayudar a la sociedad a identificar sus problemas y potencialidades, contribuye a la humanización del estudiante y a su capacitación para el mundo del trabajo. Además, en la práctica investigativo-laboral sólo pueden hacerse significativos y útiles para el estudiante los problemas que aparecen en las situaciones complejas e irrepetibles del centro de trabajo y la comunidad, por tanto las soluciones de los mismos surgen de la investigación en el contexto donde se desarrolla su acción, es decir, en la práctica investigativo-laboral que se desarrolla en las empresas debe concebirse la solución de problemas reales debido porque cuando el estudiante resuelve problemas de la producción o los servicios, también se desarrolla a sí mismo.

Se considera que las prácticas laborales o pre- profesionales son las actividades de fortalecimiento y puesta en práctica de los conocimientos y habilidades recibidas en las instituciones docentes que realizan los estudiantes de diferentes especialidades y carreras en los futuros contextos de actuación. Ellas se desarrollan durante un tiempo determinado a lo largo de los estudios, están directamente relacionadas con su formación profesional y planificadas, organizadas y controladas por la institución formadora. Su fin es esencialmente formativo y educativo.

Es importante tener en cuenta que el desarrollo de la práctica laboral investigativa propicia:

La socialización del estudiante de práctica con el entorno laboral (cómo conducirse, cuáles son las normas de comportamiento, la disciplina, las relaciones laborales, los principales procesos que se desarrollan, la implicación de todos los trabajadores para lograr un fin común.)

Motivación por desarrollar su vínculo laboral en esa misma empresa o institución, de ser continuador y parte del prestigio de ese centro. 
Familiarizarse con las tecnologías y exigencias reales, que enriquecen lo aprendido en la universidad.

Realizar tareas prácticas en correspondencia con la profesión.

Siempre será una excelente decisión el desarrollo de las prácticas laborales (en entidades, empresas, organismos e instituciones) como parte del plan de estudio de las diferentes carreras, ella propicia el vínculo teoría - práctica. Ella propicia que los estudiantes universitarios adquieran vivencias profesionales, se vinculen a proyectos y procesos laborales, aprendan de los más experimentados, constituyendo de hecho, una experiencia invaluable.

Garantizar que las prácticas laborales sean exitosas garantiza un elevado nivel de satisfacción de los alumnos por su carrera, por el lugar donde realizaron su práctica y especialmente por la universidad que propició su formación.

Sin embargo, en el desarrollo de la práctica laboral investigativa inciden de manera negativa los siguientes aspectos:

- $\quad$ Poco conocimiento por parte de la Universidad de los procesos productivos y sociales que se desarrollan en empresas e instituciones y por otro lado las empresas e instituciones tampoco tienen suficiente conocimiento de las particularidades del proceso formativo de los futuros profesionales que se desarrolla en las Universidades.

Durante la práctica laboral predomina el carácter reproductivo de las evaluaciones, se subvaloran los ejercicios y tareas de carácter práctico y que tengan como elemento esencial la creatividad y la independencia delestudiante.

- $\quad$ No siempre se planifica un adecuada guía metodológica ni un plan de práctica que propicie el desarrollo de tareas de complejidad creciente, que exijan aprendizajes desarrolladores, orientando tareas rutinarias y carentes de interés.

Insuficiente supervisión, control y evaluación de la práctica por parte de tutores/especialistas y profesores de la universidad.

- $\quad$ Algunos organismos, empresas e instituciones consideran que la atención al estudiante de práctica laboral es un gasto, una pérdida de tiempo y de recursos financieros y materiales, y no perciben el beneficio que puede reportarle este vínculo con la Universidad. Sin embargo, una vez graduados tienen que invertir recursos para la recalificación, la actualización y adiestramiento del egresado porque es vital para el éxito de la producción y los servicios y la Universidad no egresó el profesional exigido.

- $\quad$ No se asignan los mejores profesionales para atención a los practicantes.

- No siempre se coordinan con antelación ni de manera adecuada las prácticas laborales, trayendo como consecuencia la desatención por parte de los especialistas y directivos de las UD/ELB, el desinterés y la desmotivación por parte del estudiante.

Atender con prioridad el desarrollo de la práctica laboral investigativa-como expre- 
sión del principio marxista y martiano de vinculación de la teoría con la práctica, del estudio con el trabajo, significa avanzar desde el pregrado en el desarrollo de la profesionalización, en tanto en ella se propicia el desarrollo de modos de actuación acorde a la profesión que se estudia, se desarrollan valores morales, la ética de la profesión se aprende a comprender y a buscar soluciones interdisciplinarias a los diferentes problemas profesionales, se amplían, profundizan, actualizan y contextualizan los conocimientos, se desarrollan habilidades. Ella debe propiciar que se adquieran vivencias positivas sobre la profesión, que se familiaricen y resuelvan problemas de la práctica, por tanto, existe una relación recíproca.

Por tanto, se hace necesario que se logre una implicación real y efectiva por ambas partes: que la universidad se interese más por los procesos productivos, educativos y de salud, la capacitación, la solución de las problemáticas de los organismos, empresas e instituciones y que estos se impliquen más con sus recursos materiales, humanos y financieros en la formación del futuro profesional, que en un plazo relativamente breve estará en sus centros. Deben comprender que esta atención esmerada que realizan es una inversión, para en un futuro obtener mejores resultados.

Implica, por tanto, que los vínculos y la colaboración propicien:

1. Mejorar los resultados de las universidades y de los centros donde se insertan los estudiantes, de los profesores, de los estudiantes, de los trabajadores de los centros laborales y también las relaciones interinstitucionales.

2. La formación de una responsabilidad que debe ser asumida por ambas partes, y que debe incluir el pregrado, la capacitación, el postgrado y las investigaciones que los beneficien mutuamente.

3. El vínculo estrecho y coordinado. La práctica laboral investigativa no puede ser espontánea, sino planificada, organizada, controlada y evaluada y conciliada con las UD/ELB que recibirán a los estudiantes, de manera que se aseguren por ambas partes los recursos humanos y materiales necesarios para el desarrollo exitoso de la misma.

4. Necesidad de establecer convenios, en los que queden precisados los deberes y tareas de cada una de las partes, como manifestación de la voluntad de ambas partes de trabajar conjuntamente en la formación del profesional.

Con la relación universidad- empresa se logra:

La solución de problemas profesionales reales en la empresa o institución a través del trabajo científico estudiantil y de investigaciones realizadas por profesionales de la universidad y de las empresas.

Responsabilidad compartida en la formación del profesional, que incluye la preparación de los especialistas/tutores, su categorización, facilidades para el empleo de los laboratorios, talleres, tecnologías, bibliografías, etc.

La relación formación inicial del profesional adiestramiento y postgrado. 
La solución interdisciplinaria de los problemas profesionales de las empresas, solución a la que contribuyen los estudiantes de las diferentes carreras insertadas en esa empresa o institución.

- $\quad$ Aprendizaje mutuo y bilateral: los estudiantes aprenden de los especialistas y estos de los estudiantes. sitarios.

Favorecer que en la empresa o institución confluyan todos los procesos univer-

Conferir mayor pertinencia a los procesos académicos y de investigación.

- El incremento y actualización de los conocimientos y habilidades de los profesionales de las empresas/instituciones y de lasuniversidades.

- $\quad$ Proporcionar a los estudiantes conocimientos, vivencias, experiencias, conocimientos, habilidades y competencias profesionales.

- Que las empresas/instituciones reciban asesoría y asistencia para la solución de sus problemas, acceden a una información actualizada y especializada, capacitación y postgrado para su personal.

La importancia de la relación de la universidad con las empresas aparece refrendada en los acuerdos e informes de diferentes foros de organismos como la Unesco y la CEPAL. Se plantea por ejemplo que existen diversos factores que pueden facilitar la creación y el fortalecimiento de la interrelación entre las universidades y las empresas (elementos de convergencia) y otros que, por el contrario, pueden dificultar o entorpecer dicha vinculación (elementos de divergencia).

- $\quad$ Elementos de convergencia: las universidades y las empresas son actores primarios en los procesos de innovación y ambos poseen habilidades y capacidades científicas y tecnológicas fundamentales para la generación de conocimiento, que muchas veces resultan complementarias.

- Elementos de divergencia: a pesar de compartir la particularidad de desarrollar y utilizar capacidades para la generación de conocimiento científico-tecnológico, la universidad y la empresa llevan adelante esta tarea con competencias, finalidades y criterios muy distintos.

Por tanto, la creación de vínculos entre universidades y empresas debe desarrollarse según la noción de especialización complementaria: generar y fortalecer los factores de convergencia entre ellas, es decir, sus respectivas capacidades científicas y tecnológicas y, al mismo tiempo, reducir los elementos de divergencia, sin que esto implique eliminar las especificidades, objetivos y misiones de cada una.

Este vínculo se manifiesta de diferentes formas y se establece a través de diferentes canales, obteniéndose diferentes beneficios. Se resaltan los siguientes:

- flujos de recursos humanos (por ejemplo, las prácticas de los estudiantes en empresas o la contratación de nuevos profesionales),

- las redes informales entre profesionales, 
- las actividades de difusión del conocimiento (conferencias, elaboración de publicaciones),

- los proyectos específicos,

- $\quad$ los servicios de asesoramiento y apoyo tecnológico (asistencia técnica, equipo técnico).

- $\quad$ el licenciamiento de tecnología (patentes, oficinas de transferencia tecnológi cas de las universidades),

- $\quad$ la creación de centros mixtos o empresas de base tecnológica.

En el desarrollo exitoso de la práctica laboral investigativa un papel esencial lo desempeña el especialista/tutor de los estudiantes que labora en las unidades docentes y entidades laborales de base.

Las unidades docentes y las entidades laborales de base se convierten en el centro integrador y promotor de las potencialidades científicas y laborales en la formación del profesional; en ellas el especialista/ tutor desempeña un papel importante y decisivo y la tutoría debe caracterizarse por la flexibilidad y atención diferenciada a cada uno de los tutorados de acuerdo con sus características, intereses, conocimientos, capacidades, nivel de dificultades y avances, esto es: atender las características personales de cada estudiante practicante de manera individualizada, pero dentro del contexto colectivo.

La revisión bibliográfica realizada permitió constatar que desde tiempos remotos han existido "maestros" que han conducido a uno o varios "aprendices" o discípulos por el camino del conocimiento y han impregnado la personalidad de estos con su ejemplo personal, saturado de valores como la constancia, la entrega al trabajo, al estudio y la investigación, la responsabilidad, honestidad, ejemplos muy conocidos son: Aristóteles, Sócrates, Platón, Juan Jacobo Rousseau, Simón Rodríguez, Rafael María Mendive. Así pudieran citarse numerosos ejemplos de mentores, tutores y maestros que han guiado el aprendizaje de sus discípulos oportunamente a través del tiempo, porque la tutoría como función educativa aparece a comienzos del siglo $\mathrm{XI}$ en las universidades.

El tutor era un profesor que ejercía la función de tutela formativa, asegurando el estilo universitario, convirtiéndose en el garante de la universidad científica ante los estudiantes cuya formación tenía encomendada.

En diferentes partes del mundo este profesional es identificado como: un paradigma de la educación;un profesional que se ajusta a los requerimientos exigidos por el régimen tutorial y que ha recibido un entrenamiento para realizar con propiedad su rol ; como el profesional que adquiere formalmente un compromiso frente a una institución y para el tutorado . 
De todo lo anterior se desprende que el tutor debe caracterizarse por poseer cualidades humanas (amistad, respeto, exigencia, justicia, humildad, vitalidad, empatía, madurez, responsabilidad, paciencia y optimismo, rectitud, preocupación por los estudiantes bajo su responsabilidad), científicas y técnicas .Otro elemento importante que debe considerarse en la planificación, organización, ejecución, control y evaluación de la práctica laboral es la preparación de los diferentes actores principales: los estudiantes, los especialistas/ tutores y directivos de las UD/ELB y los profesores de la universidad responsabilizados con la tarea.

En el caso particular de los estudiantes es importante que ellos conozcan y concienticen los siguientes aspectos:

1. Cumplir en la forma planificada el desarrollo y evaluación de la práctica laboral.

2. El clima organizacional y comunicacional, la forma de vestir, los compromisos, el régimen de vida de unidad docente o entidad laboral de base donde realizan la práctica laboral investigativa, es diferente al de la universidad, por tanto, deben ser respetuosos y cooperar en las tareas.

3. Deben establecer relaciones cordiales, amables con los directivos, especialistas y trabajadores, pero siempre utilizando un lenguaje formal y respetuoso. En, es decir, una comunicación respetuosa y asertiva.

4. Cumplir con los horarios establecidos y con los compromisos, tareas o proyectos encomendados. En los casos en que el practicante requiera atender un compromiso importante a nivel académico o por asuntos personales, debe hablar con su jefe inmediato y acordar cómo es el proceso formal para solicitar el permiso y bajo qué condiciones se llevará a cabo.

5. Debe ser proactivo en las actividades de su práctica laboral, imprimirles entusiasmo, calidad, interés, compromiso, flexible y mantener buena disposición para resolverlas.

6. Debe ser recursivo y creativo. En caso de no tener los conocimientos necesarios para resolver las tareas buscar la información necesaria a través de diferentes vías. Evitar realizar un trabajo que carezca de calidad y que afecte tanto a suformación como a la UD/ELB.

En correspondencia con el análisis realizado se propone una estrategia pedagógica para perfeccionar la integración y cooperación entre la universidad y los OACE que potencie el desarrollo de la práctica laboral investigativa.

Se concibe la estrategia como acciones que se llevan a cabo para alcanzar determinado objetivo social, acciones secuenciales e interrelacionadas que, partiendo de un estado inicial, y considerando los objetivos propuestos, permite dirigir el desarrollo de formación inicial del profesional durante la práctica laboral investigativa en las ELB/ UD de los diferentes OACE. Se caracteriza por ser flexible, orientadora, participativa y dinámica 
Objetivo de la estrategia: Perfeccionar el desarrollo de la práctica laboral investigativa, a partir de la integración y cooperación entre la universidad y los OACE.

Primera etapa: Planeación.

- $\quad$ Proyección a nivel de Facultad y carrera del desarrollo de la práctica laboral investigativa: se elaboran las guías metodológicas (las tareas que se incluyan deben tomar en consideración la necesaria relación entre lo académico, lo laboral investigativo y lo extensionista, así como el carácter productivo y vivencial de las mismas) para estudiantes y tutores/especialistas y el Plan de práctica por años. Se designan los profesores que atenderán la práctica laboral investigativa (PLI).

- $\quad$ Se realizan las coordinaciones iniciales con los OACE para determinar - en correspondencia con los objetivos de la práctica laboral, la cantidad y lugar de residencia de los estudiantes - las UD/ELB que posean las mejores condiciones para el desarrollo de la PLI.

- Se realiza un diagnóstico de las UD/ELB para precisar sus potencialidades, fortalezas, debilidades y necesidades y posibles tutores/especialistas.

- Intercambios de la dirección de la universidad y sus facultades con las direcciones de los OACE, UD/ ELB, para precisar los principales aspectos que serán convenidos.

- $\quad$ Desarrollo de talleres metodológicos y de socialización para elaborar los materiales y medios didácticos que se emplearan en la capacitación de los directivos, tutores /especialistas de los OACE y ELB/UD, ejemplo plegable sobre los fundamentos legales de la PLI.

- $\quad$ Capacitación de los responsables de la PLI y capacitadores de las UD/ELB sobre los fundamentos legales de la PLI.

- $\quad$ Categorización de los tutores/especialistas.

Este proceso debe realizarse con un año de anterioridad (preferentemente en el primer trimestre).

Segunda etapa: Concertación.

- $\quad$ Firma y/o actualización de los convenios de la práctica laboral investigativa.

- La rectora de la universidad firma un convenio marco con los directivos de los OACE y otras instituciones de importancia en el territorio y de otras provincias en las que existan instituciones y empresas afines al perfil profesional de las carreras que se estudian en la universidad.

-Los decanos de las facultades firman los convenios con las UD/ELB. Se precisan las tareas a desarrollar de conjunto, objetivos, fechas y responsables y las formas en que se desarrollará la práctica laboral investigativa.

A nivel de universidad, facultad, OACE y UD/ELB debe quedar un ejemplar del convenio firmado. 
Tercera etapa: Ejecución.

- $\quad$ El profesor designado por la carrera realiza el proceso de entrega pedagógica y presentación del estudiante a la UD/ELB seleccionada (explicando y entregando la guía metodológica y el Plan de práctica laboral investigativa).

- Discusión y análisis de los estudiantes, directivos y especialistas/tutores de la UD/ELB del Reglamento de la Práctica laboral investigativa.

- Desarrollo de la PLI bajo la supervisión del tutor/especialista y del profesor de la universidad. Durante el desarrollo de la misma debe establecerse un fuerte y sistemático vínculo de las carreras con la empresa o institución donde está ubicado el estudiante para comprobar el desarrollo del estudiante y el cumplimiento de las actividades planificadas.

- Desarrollo de actividades conjuntas en ambas instituciones para propiciar el conocimiento de los procesos que se desarrollan (productivos - sociales y formativos).

- $\quad$ Participación de los profesionales de la UD/ELB en las actividades metodológicas y en la impartición de docencia en la universidad y los profesores de la universidad se incorporan a los procesos que se desarrollan en las UD/ELB.

- Se continúa desarrollando la capacitación a los especialistas/tutores.

- Recorrido de los directivos de la universidad, facultades y carreras por las ELB/UD para comprobar el desarrollo de la PLI y precisar la integralidad del trabajo cooperado universidad-empresa (Tutoría, capacitación, aseguramiento de locales, laboratorios, espacios productivos y de servicios).

Cuarta etapa: Control y evaluación.

Se realiza durante todo el proceso de práctica laboral investigativa. Su objetivo es constatar el cumplimiento de los objetivos propuestos.

- La evaluación final del estudiante se realiza en correspondencia con las normas establecidas por cada una de las carreras y con la participación de especialistas de las empresas. Deben garantizar el desarrollo de la independencia, la creatividad y la integración de los conocimientos por parte de los estudiantes.

- Los directivos de las UD/ELB y de los OACE elaboran un informe del desarrollo de la práctica laboral investigativa precisando las críticas y sugerencias a la formación del profesional, qué le falta en su Plan de estudio, cómo mejorar su formación.

- $\quad$ Se precisan las vías para que el especialista/tutor se incorpore a la universidad y participe en actividades metodológicas y en la impartición de asignaturas del currículo propio/optativo.

- $\quad$ Con el propósito de valorar la marcha de la vinculación universidad - OACE y de establecer las pautas para la nueva etapa, se desarrollan reuniones de trabajo en los meses de octubre, febrero y junio. 
- $\quad$ Desarrollo de talleres metodológicos para evaluar los resultados de la PLI, con la participación de los directivos de los OACE.

La aplicación de la estrategia en el curso escolar 2015- 2016 propició:

a) Vínculos estables de trabajo, cooperación y colaboración con los OACE.

b) La participación de directivos y profesionales de las Unidades Docentes y Entidades Laborales en el proceso de acreditación de las carreras.

c) Eficiente respuesta de los organismos para la atención de los estudiantes en condiciones de contingencia (sismos y huracanes).

d) Aumenta en todas las facultades la calidad, el número y la diversidad de asignaturas del currículo optativo/electivo que responden a las necesidades de la profesión y el territorio, con la presencia de profesionales de los OACE.

e) Incremento de la motivación de los estudiantes por desarrollar su vínculo laboral en esa misma empresa o institución, de ser continuador y parte del prestigio de ese centro.

f) Incremento del nivel de satisfacción de los alumnos con respecto a la contribución de la práctica laboral en la solución de los problemas más generales de la profesión y al tratamiento a los valores y normas de conducta universitaria en la práctica laboral.

g) La solución de problemas profesionales reales en la empresa o institución a través del trabajo científico estudiantil y de investigaciones realizadas por profesionales de la universidad y de las empresas.

h) Incremento de la preparación de los especialistas/tutores, su categorización, y de las facilidades para el empleo de los laboratorios, talleres, tecnologías, bibliografías, etc.

i) Mayor acercamiento de la universidad a las empresas/instituciones a través de asesoría y asistencia para la solución de sus problemas, acceso a una información actualizada y especializada, capacitación y postgrado para su personal.

j) Actualización del currículo de las carreras con la incorporación de cursosoptativo/electivos en correspondencia con las necesidades y sugerencias de los OACE para favorecer el egresado que se necesita.

k) Incremento del interés de los directivos de los OACE por profundizar los vínculos con la universidad (solicitud de cursos y maestrías, de categorización de los especialistas, de propuestas de cursos optativos/electivos y participación en actividades metodológicas sobre PLI en las carreras).

\section{CONCLUSIONES}

El perfeccionamiento de la práctica laboral investigativa constituye una exigencia en el empeño de garantizar la formación del profesional que exige la sociedad y por tanto su diseño debe realizarse sobre la base de la concertación de acuerdos entre la universidad (como centro formador) y los OACE (representados por UD/ELB e instituciones) como centros empleadores en los cuales desarrollarán su futuro laboral. 
Las unidades docentes y las entidades laborales de base se convierten en el centro integrador y promotor de las potencialidades científicas y laborales en la formación del profesional; por tanto, otro elemento importante para perfeccionar la PLI en las ELB/UD es el conocimiento que tienen que tener cada uno de los actores principales de este proceso.

\section{REFERENCIAS BIBLIOGRÁFICAS}

Alarcón, R. (2016). Conferencia inaugural Universidad 2016. Palacio de las Convenciones. La Habana. En digital.

Asín, M. (2011). El tutor del docente en formación, figura necesaria en la forma- ción inicial del profesional de la educación. Revista Electrónica Cuadernos de Educación y Desarrollo. ISSN 1989-4155. Indexada en IDEAS-RePEc. www. eumed. net/rev/ced, publicado en el No 27, mayo 2011 y alojada en http:// www. eumed. net/rev/ced/2//cmb.htm.

Castro, F. (1980). El estudio, el trabajo y la formación de la juventud. Selección de textos. Editora Política. La Habana.

Cepal. (2010). Espacios iberoamericanos: vínculos entre universidades y empresas para el desarrollo tecnológico. En digital.

Comenius, J. (1998). Didáctica Magna. 8va edición. Editorial Porrúa, México.

Díaz, T. (2010). Fundamentos pedagógicos y didácticos de la Educación Superior. Curso pre- evento de Universidad 2010. Palacio de las Convenciones. La Habana.

Herrera, J.L. (2006). El vínculo universidad-empresa en la formación de los profesionales universitarios. Revista Electrónica Actualidades Investigativas en Educación, vol. 6, núm. 2, mayo-agosto, 2006. P.12. Disponible en http//: www. Redalyc.

Martí, J. (1975). La Escuela de Artes y Oficios en Honduras. En Obras Completas. Tomo 8. Editorial Ciencias Sociales. La Habana.

Martí, J. (1978). Trabajo Manual en las Escuelas. En Obras Escogidas en tres tomos. Editora Política. La Habana.

Ministerio de Educación de Cuba. (2007) Reglamento del Trabajo Docente - Meto dológico.

Resolución No. 210/2007. Ministerio de Educación Superior de Cuba. (1985) Reslución 89/85. 